\title{
Canadian consensus recommendations for the optimal use of enfuvirtide in HIV/AIDS patients
}

\author{
Anita Rachlis MD MEd FRCPC ${ }^{1}$, Jonathan Angel MD², Marianne Harris MD CCFP ${ }^{3}$, Richard Lalonde MD FRCPC ${ }^{4}$, \\ Fiona Smaill MB ChB ${ }^{5}$, Cecile Tremblay MD FRCPC ${ }^{6}$, Chris Tsoukas MD ${ }^{7}$, Sharon Walmsley MD ${ }^{8}$
}

\begin{abstract}
A Rachlis, J Angel, M Harris, et al. Canadian consensus recommendations for the optimal use of enfuvirtide in HIV/AIDS patients. Can J Infect Dis Med Microbiol 2006;17(3):155-163.
\end{abstract}

BACKGROUND AND OBJECTIVES: An eight-member group consisting of Canadian infectious disease and immunology specialists and a family physician with significant experience in HIV management was convened to update existing recommendations, specifically intended for use by Canadian HIV-treating physicians, on the appropriate use of enfuvirtide in HIV/AIDS patients with resistance to other antiretroviral drugs.

METHODS: Evidence from the literature and expert opinions of the group members formed the basis of the guidelines. Comments on the draft guidelines were obtained from other physicians across Canada with HIV expertise. The final guidelines represent the group's consensus agreement.

RESULTS AND CONCLUSIONS: The recommendations were developed to guide physicians in optimal practices in patient selection for enfuvirtide treatment and subsequent patient management. The issues considered include positive predictors of response to enfuvirtide, stage of disease, optimization of the background regimen, early indicators of enfuvirtide response, and patient education and support.

\section{Les recommandations consensuelles canadiennes pour l'utilisation optimale d'enfuvirtide chez les patients atteints du VIH-sida}

HISTORIQUE ET OBJECTIFS : Un groupe de huit personnes composé de spécialistes canadiens de l'infectiologie et de l'immunologie et d'un médecin de famille ayant une vaste expérience de la prise en charge du VIH s'est réuni pour mettre à jour les recommandations existantes, conçues notamment pour les médecins canadiens traitant le VIH, au sujet de l'usage convenable d'enfuvirtide chez les patients atteints du VIH-sida résistants à d'autres antirétroviraux.

MÉTHODOLOGIE : Les données probantes tirées des publications et les avis spécialisés des membres du groupe ont formé le fondement des lignes directrices. D'autres médecins du Canada possédant des connaissances spécialisées en matière de VIH ont commenté la version provisoire des lignes directrices. Les lignes directrices définitives représentent le consensus du groupe.

RÉSULTATS ET CONCLUSIONS : Les recommandations ont été élaborées pour orienter les médecins dans des pratiques optimales pour sélectionner des patients qui recevront un traitement à l'enfuvirtide et une prise en charge subséquente. Les enjeux abordés sont les prédicteurs d'une réaction positive à l'enfuvirtide, le stade de la maladie, l'optimisation de la posologie de fond, les indicateurs précoces d'une réaction à l'enfuvirtide ainsi que l'éducation et le soutien des patients.

Key Words: AIDS; HIV; Recommendations; Treatment

\section{DEVELOPMENT PROCESS FOR GUIDELINES} The purpose of the present guidelines is to update existing recommendations (1) on the appropriate use of enfuvirtide in HIV/AIDS patients with resistance to other antiretroviral drugs. An eight-member group of infectious disease and immunology opinion leaders from across Canada and a family physician with significant experience in HIV management was convened by Roche Canada in March 2005. The group identified areas relevant to the use of the enfuvirtide in advance as topics for the guidelines. Group members identified the relevant literature by search and review, and presented their findings to the group for discussion. The expert opinions of the group members were included as evidence. The strength of each recommendation was categorized according to a standard rating system (Table 1). Comments on the draft guidelines were obtained from the group, as well as from primary care physicians and other specialists across Canada with HIV expertise. The final guidelines represent the group's consensus agreement.

${ }^{1}$ Division of Infectious Diseases, Department of Medicine, Sunnybrook Health Sciences Centre, University of Toronto, Toronto; ${ }^{2}$ Division of

Infectious Diseases, Department of Medicine, Ottawa Hospital and University of Ottawa, Ottawa, Ontario; ${ }^{3}$ AIDS Research Program,

St Paul's Hospital, Vancouver, British Columbia; ${ }^{4}$ Immunodeficiency Service and Division of Infectious Diseases, McGill University Health

Centre, Montreal, Quebec; ${ }^{5}$ Department of Pathology and Molecular Medicine, Faculty of Health Sciences, McMaster University, Hamilton,

Ontario; ${ }^{6}$ UHRESS, Centre Hospitalier de l'Université de Montréal; ${ }^{7}$ Department of Medicine, McGill University Health Centre, Montreal,

Quebec; ${ }^{8}$ Division of Infectious Diseases, Department of Medicine, Toronto General Hospital, University of Toronto, Toronto, Ontario

Correspondence: Dr Anita Rachlis, A226, Sunnybrook Health Sciences Centre, 2075 Bayview Avenue, Toronto, Ontario M4N 3 M5.

Telephone 416-480-4689, fax 416-480-5808, e-mail a.rachlis@utoronto.ca

Received for publication November 28, 2005. Accepted April 28, 2006 


\section{TABLE 1 \\ Categories reflecting the strength of each recommendation for or against use and the quality of evidence on which the recommendations are based}

\begin{tabular}{ll}
\hline Category & Definition \\
\hline Strength of recommendation \\
A & Good evidence in support of a recommendation for use \\
B & Moderate evidence to support a recommendation for use \\
C & Poor evidence to support a recommendation for or against use \\
D & Moderate evidence to support a recommendation against use \\
E & Good evidence to support a recommendation against use \\
Grade & Definition
\end{tabular}

Quality of evidence
Evidence from at least one properly randomized, controlled
trial
Evidence from at least one well-designed clinical trial without
randomization, from cohort or case-controlled analytic studies
(preferably from more than one centre), from multiple
time-series studies, or from dramatic results in uncontrolled
experiments
Evidence from opinions of respected authorities, based on
clinical experience, descriptive studies, or reports of expert
committees

Data from reference 74. Reprinted with permission from The University of Chicago Press

The recommendations contained in these guidelines are intended for use by health care providers who treat patients with HIV infection. These recommendations are not a substitute for the judgement of a physician experienced in treating these patients.

\section{INTRODUCTION}

The effective management of people living with HIV improves clinical outcomes $(2,3)$. The current treatment standard is to initiate antiretroviral combination therapy with two nucleoside reverse transcriptase inhibitors (NRTI) and either a protease inhibitor (PI) (boosted with ritonavir, or unboosted) or a nonNRTI $(4,5)$. With this approach, most patients can achieve sustained virological suppression to less than 50 copies $/ \mathrm{mL}$ and substantially increase their CD4 cell counts. Furthermore, the prevalence of drug resistance has declined (6-8).

Despite these advances, treatment failure still occurs and drug resistance remains an important clinical problem (6-8). Data from 64 trials in antiretroviral-naive patients between 1994 and 2004 (9) indicated that treatment failed in approximately $36 \%$ to $56 \%$ of patients by week 48 . After the introduction of highly active antiretroviral therapy (HAART), many patients acquired multidrug-resistant viral strains, and patients with drug-resistant virus have a greater risk of disease progression and death (10). Therefore, new agents continue to be important in the management of patients with HIV/AIDS.

\section{THE MANAGEMENT OF PATIENTS WITH MULTIDRUG-RESISTANT HIV}

A physician caring for a patient on HAART with a detectable viral load and multidrug-resistant HIV has several options: first, a switch to a new regimen with as many active agents as possible (based on viral resistance genotyping); second, megaor giga-HAART regimens (salvage regimens that contain six or more antiretroviral drugs, some of which may be partially active); third, treatment interruption before initiation of a new salvage regimen (ie, first or second option); or fourth, continuing a failing regimen or switching to a partially suppressive regimen in an attempt to maintain poor viral fitness and reduced viral replication while waiting until new, active treatment options are available. These options usually apply to treatmentexperienced patients with virological failure, but treatmentnaive patients may also exhibit multidrug resistance, although rarely $(11,12)$.

There are a number of issues to consider in selecting one of these options: antiretroviral treatment history, taking into account adherence and toxicities, in concert with resistance testing results $(13,14)$; comorbidities that may affect treatment response, adherence and drug selection (eg, hepatitis B and C virus coinfection, cardiovascular disease and diabetes); current and nadir CD4 cell count and fraction; viral load and trends; agents currently available and those likely to be available within two years; and, therapeutic options if this combination fails.

Although suppression of viral replication to less than 50 copies $/ \mathrm{mL}$ is the ideal goal of therapy, it may not always be achievable. In patients with advanced disease and drug-resistant virus, immunological stability and the prevention of clinical disease progression may be more realistic goals of therapy than is complete viral suppression (15). However, it is important to not prematurely reduce expectations about achieving virological suppression when viable treatment options are still available (16).

\section{OVERVIEW OF ENFUVIRTIDE}

Enfuvirtide, also known as T-20, is the first member of a new class of antiretroviral drugs called fusion inhibitors. It is a 36-amino acid synthetic peptide inhibitor of gp41-mediated fusion between HIV and the membrane of the target CD4 lymphocyte (17). Because it is a large peptide, enfuvirtide must be administered by subcutaneous injection at a dose of $90 \mathrm{mg}$ twice daily (18). The extracellular mode of action and unique antiviral target results in several benefits, including the absence of cross-resistance with other antiretroviral agents, as well as decreased risk of systemic toxicity and drug interactions $(18,19)$. Treatment response to enfuvirtide is not affected by viral tropism for CCR5 and CXCR4 coreceptors (17).

\section{Enfuvirtide efficacy}

The importance of enfuvirtide in the management of treatmentexperienced patients was initially demonstrated in the T-20 versus Optimized Regimen Only 1 (TORO 1) study and the TORO 2 study $(20,21)$. Treatment-experienced adult patients from Europe, Australia and the Americas with at least three or six months previous experience with NRTI, non-NRTI and one to two PIs, and/or resistance to each of these classes of agents, were enrolled in these studies $(n=995)$. Patients were randomly assigned to treatment for 48 weeks with either enfuvirtide plus optimized background therapy or optimized background alone, followed by a 48-week optional extension period of enfuvirtide plus optimized background therapy (52\% of patients entered the extension). Optimized background selection was guided by genotypic and phenotypic resistance testing, as well as previous antiretroviral treatment and tolerance history.

The key efficacy results from the combined intent-to-treat meta-analysis of the TORO studies at 48 weeks of treatment 


\section{TABLE 2}

Summary of key efficacy results from the T-20 versus Optimized Regimen Only 1 (TORO 1) and TORO 2 studies* (22)

\begin{tabular}{|c|c|c|c|}
\hline Parameter & Enfuvirtide plus optimized background & Optimized background alone & $\mathbf{P}$ \\
\hline $\begin{array}{l}\text { Least squares mean change in plasma HIV RNA from } \\
\text { baseline }\left(\log _{10} \text { copies } / \mathrm{mL}\right)\end{array}$ & -1.48 & -0.63 & $<0.0001$ \\
\hline Viral load $<400$ copies $/ \mathrm{mL}$ (\% of patients) & 30.4 & 12.0 & $<0.0001$ \\
\hline Viral load $<50$ copies $/ \mathrm{mL}$ ( $\%$ of patients) & 18.3 & 7.8 & $<0.0001$ \\
\hline Median time to virological failure (weeks) & 32 & 11 & $<0.0001$ \\
\hline Least squares mean CD4 count increase (cells $/ \mu \mathrm{L}$ ) & +91 & +45 & $<0.0001$ \\
\hline \multicolumn{4}{|l|}{ • Higher CD4 cell counts } \\
\hline \multicolumn{4}{|l|}{ - Lower viral loads } \\
\hline \multicolumn{4}{|l|}{ - Less treatment experience } \\
\hline - Resistance to fewer agents & & & \\
\hline
\end{tabular}

*These data are from the intent-to-treat population, with the patients who switched to enfuvirtide therapy excluded from the analysis

(22) are summarized in Table 2. Long-term follow-up of these patients to 96 weeks of treatment showed durability in patient response (23). The majority of patients who were responders (virological or immunological) at weeks 24 and 48 continued to respond at 96 weeks; $26.5 \%$ continued to have HIV RNA levels under 400 copies $/ \mathrm{mL}$ and $31.5 \%$ continued to have a CD4 count increase of 100 cells/ $\mu \mathrm{L}$ or higher (intent-to-treat analysis).

In the TORO trials, patients who failed therapy with the optimized background therapy alone (control arm) had the opportunity to switch after eight weeks to a new, optimized background therapy that included enfuvirtide. Therefore, it was possible to evaluate the impact of the drug when used later in the treatment course. In the patients in whom enfuvirtide was introduced later, both the decline in viral load and the increase in CD4 count were substantially less (23), likely due to the acquisition of new resistance and the re-emergence of pre-existing resistance due to lack of complete virological suppression (24).

Studies of newer protease inhibitors, such as tipranavir and darunavir (TMC 114), provide further evidence of the efficacy of enfuvirtide in treatment-experienced patients. In the Randomized Evaluation of Strategic Intervention in MultiDrug ReSistant Patients with Tipranavir 1 (RESIST-1) trial (25), a 24-week viral load reduction to under 400 copies/mL was seen in $47 \%$ of patients treated with an optimized background regimen plus tipranavir/ritonavir (TPV/r) plus enfuvirtide versus $34.7 \%$ who received an optimized background regimen plus TPV/r only. Similarly, $22 \%$ of patients that received enfuvirtide had viral load suppression in the comparator group (an optimized regimen that included a ritonavirboosted PI) versus $16.5 \%$ of patients in the comparator group that did not receive enfuvirtide. In the combined RESIST-1 and RESIST-2 trials, $35.8 \%$ of patients treated with an optimized background regimen plus TPV/r plus enfuvirtide had viral load reduction to less than 50 copies/mL at 48 weeks versus $14.4 \%$ who received an optimized background regimen plus comparator-boosted PIs plus enfuvirtide $(25,26)$. At week 24 of the Performance Of TMC114/r When Evaluated in tripleclass-experienced patients with PI Resistance 1 (POWER 1) and POWER 2 studies, 63\% and 64\% (respectively) of enfuvirtide-naive patients who received enfuvirtide plus darunavir/ritonavir (TMC 114/r) as part of their regimen achieved viral load reductions of less than 50 copies/mL, versus
$56 \%$ and $30 \%$ (respectively) of patients who received the same regimen without enfuvirtide $(27,28)$.

\section{Enfuvirtide safety}

Enfuvirtide is generally well tolerated. In clinical trials, most adverse events have been mild to moderate in intensity. No exacerbation of other common antiretroviral toxicities by enfuvirtide has been observed. Treatment discontinuations for safety reasons occurred in $14.0 \%$ of patients in the TORO studies after 48 weeks $-4.4 \%$ for injection site reactions, $8.9 \%$ for adverse events of any cause, $0.3 \%$ for laboratory anomalies and $0.5 \%$ for deaths (29).

The most common adverse event associated with enfuvirtide is injection site reaction, characterized by tender erythematous nodules at the injection site that may persist. For the majority of patients, injection site reactions are mild and tolerable, with few individuals requiring drug discontinuation or analgesics to control them $(23,29)$. Although injection site reactions occurred in $98.3 \%$ of enfuvirtide-treated patients in the TORO trials in the initial 48-week treatment period, these reactions limited treatment in less than $5 \%$ of patients (29). The incidence of injection site infections, including abscesses and cellulitis, was 2.4 per 100 patient-years (18).

Constitutional adverse events, especially gastrointestinal events, were less common in the enfuvirtide-treated patients in the TORO studies versus those treated with optimized background alone. These events included diarrhea, nausea and fatigue. Changes in fat distribution were equally common in enfuvirtide-treated patients and in those not treated with enfuvirtide (30). Systemic hypersensitivity to enfuvirtide occurred in less than $1 \%$ of patients in the TORO trials (29).

Pneumonia was more common in the enfuvirtide-treated patients in the TORO studies $(23,29)$. The incidence of pneumonia in this group (2.7\% at week 48 and $1.2 \%$ at week 96 ) was similar to the expected range in patients with advanced HIV infection from the literature, while the optimized background alone arm of the study had a lower incidence than expected. The drug relationship of this finding has not been established, and the reason for the difference is currently unknown (29). The risk factors for pneumonia were low baseline CD4 cell count, intravenous drug use, prior or current tobacco use, and a prior history of lung disease.

Antibodies to gp41, an HIV-1 envelope glycoprotein (a fragment of which is identical to enfuvirtide), are produced 
soon after HIV infection and may affect enfuvirtide response. Data from the TORO studies showed that enfuvirtide crossreactive gp41 antibody did not affect patient safety or efficacy (31).

Recommendations for patient safety considerations during enfuvirtide therapy:

- Educate the enfuvirtide-treated patient about pneumonia symptoms and counsel him to contact his physician if symptoms appear (IIB).

- Pneumococcal immunization should be provided and repeated every five years as part of standard care of HIV patients (IIIC).

\section{Enfuvirtide resistance}

A wide range of baseline susceptibilities to enfuvirtide have been identified, but no consistent relationship to viral load response has been found (32). Mutations in amino acid positions 36 to 45 on the gp41 sequence are associated with reduced in vitro susceptibility to enfuvirtide (33-35). Data from Canadian patients failing enfuvirtide therapy indicate that mutations at G36D, V38A, V38M, N42T and N43D were most frequently associated with enfuvirtide resistance (35). Some enfuvirtide-resistant isolates have also been found to decrease replicative capacity $(17,36,37)$ and fitness (38).

CCR 5 inhibitors are currently being evaluated as antiretroviral drugs. No information is currently available on the impact of enfuvirtide resistance on response to CCR5 inhibitors.

\section{Recommendation for enfuvirtide resistance testing:}

- Resistance testing for enfuvirtide is not currently recommended (or available) (IIC).

\section{SELECTION OF PATIENTS FOR ENFUVIRTIDE THERAPY}

\section{Treatment-experienced patients}

Most of the data available for enfuvirtide support its use in treatment-experienced patients (eg, TORO [20-22], RESIST $[25,26,39,40]$ and POWER $[27,28]$ studies).

Some patients are more likely to benefit from enfuvirtide treatment. Independent positive predictive factors for virological response to enfuvirtide were identified from a logistic regression analysis of the TORO 48-week data (41):

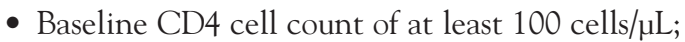

- Baseline plasma HIV-1 RNA level under $5 \log _{10}$ copies/mL;

- A history of 10 or fewer prior antiretrovirals; and,

- Two or more active antiretrovirals in optimized background regimen.

One study (41) found a positive correlation between the number of these predictive factors and response rate. In patients receiving enfuvirtide plus optimized background, $67 \%$ with all four factors had HIV-1 RNA levels under 400 copies/mL at week 48 of treatment versus 20\% with only one predictive factor (41).

Although enfuvirtide treatment is beneficial at later stages of disease (42), patients have improved outcomes with the earlier use of enfuvirtide. In the TORO studies, patients who received enfuvirtide from the start of the study had better outcomes than those who added enfuvirtide later, after virological failure with optimized background regimen alone (24). Patients with less treatment experience and more active agents in the optimized background regimen (agents given concomitantly with enfuvirtide) had a greater benefit from the inclusion of enfuvirtide in their regimens $(22,41)$. A less-than-optimal salvage regimen and failure to maximally suppress viral replication allows for the accumulation of resistance mutations, which limits future optimized background choices to be used with enfuvirtide $(43,44)$. These findings suggest that enfuvirtide should be considered earlier in the management of HIV as part of salvage regimens that include other active agents, and that it should not be reserved until late in the disease when its use may effectively be limited to monotherapy.

In pediatric patients, the pharmacokinetics, safety and efficacy of enfuvirtide are similar to those in adults, although the number of children studied to date is small $(18,45,46)$.

Issues such as toxicity, costs and the impact on the response to subsequent therapies are considerations in the use of enfuvirtide therapy.

\section{Treatment-naive patients and those with less treatment experience}

Although enfuvirtide is recommended for treatmentexperienced patients (usually those with multidrug resistance), it may have a role in certain treatment-naive patients. Although rare, treatment-naive patients can have significant drug resistance due to transmission of antiretroviral-resistant strains (13). A 2005 report from New York City (12) identified a treatment-naive patient with a highly resistant strain of HIV that had in vitro resistance to multiple classes of antiretroviral drugs but was susceptible to enfuvirtide. A finding of primary multiple class resistance may put patients in the same position as treatment-experienced patients in terms of the expected benefit of enfuvirtide treatment, but no supporting data are currently available. A recent report of a small number of patients less experienced with antiretrovirals suggested clinical benefit with the use of enfuvirtide (47).

Recommendations for the selection of patients for enfuvirtide therapy:

- Current data indicate that predictive factors, such as a baseline CD4 count of at least 100 cells/ $\mu \mathrm{L}$, baseline plasma HIV-1 RNA level under $5 \log _{10}$ copies/mL, a history of 10 or fewer prior antiretrovirals, and at least two active antiretrovirals in the treatment regimen, are useful in identifying patients in whom enfuvirtide treatment is more likely to be effective (IA).

- Although the TORO trials demonstrated the benefit of enfuvirtide in patients who were triple antiretroviral drug class-experienced (not necessarily multidrug resistant or with virological failure), its benefits may not be limited to these patients. The best response is seen when other active agents are included in the optimized background regimen, preferably from a drug class to which the patient has not been previously exposed (IA).

- Enfuvirtide may also be useful for patients who have intolerance but not necessarily resistance to other agents (IIIC). 
- Inclusion of enfuvirtide in a treatment regimen to provide another active drug class should be considered in treatment-naive patients with primary multidrug resistance (IIIB).

- Enfuvirtide is a useful addition to the treatment regimen even in late-stage disease. For patients with advanced HIV infection and no other fully active agents available, enfuvirtide may provide some benefit in combination with partially active agents (IB).

- Pediatric patients with a multidrug-resistant virus may similarly benefit from enfuvirtide treatment (IIIB).

\section{THE MANAGEMENT OF PATIENTS SELECTED FOR ENFUVIRTIDE THERAPY}

The role of treatment interruption before the initiation of enfuvirtide therapy

In the TORO studies, patients who had treatment interruptions before enrollment in the study did not have an advantage in long-term virological and immunological response over those who immediately switched to new agents (48). In a study of treatment-experienced patients that compared 24 weeks of enfuvirtide plus optimized background treatment versus 16 weeks of structured treatment interruption followed by enfuvirtide plus optimized background, no significant difference was found between treatment groups in the proportion of patients with a viral load under 75 copies/mL (53\% versus $36 \%$, respectively) (49).

Recommendation for the role of treatment interruption in enfuvirtide therapy:

- Treatment interruption before the initiation of enfuvirtide plus optimized background therapy does not improve outcome and is therefore not recommended (IE).

\section{Optimizing the background regimen}

A number of trials support the use of resistance testing to guide optimized background therapy selection (50-55). Prior resistance test results are also useful, because a resistance-related mutation that is not currently measurable by standard assays may reappear quickly when antiretroviral therapy is reintroduced (14). Expert advice to interpret the resistance test results significantly benefits the selection of optimal background therapy $(50,52)$.

The use of more active agents in the optimized background regimen, including new boosted PIs $(39,41,56)$, improves virological response to enfuvirtide and limits new mutations, therefore limiting the emergence of further drug resistance (24). In the TORO trials, the time to virological failure correlated positively with the phenotypic susceptibility score (the number of susceptible drugs and, therefore, active agents) in the background regimen $(42,57)$.

Schapiro et al (58) showed that a higher tipranavir mutation score was associated with reduced virological response in patients also treated with enfuvirtide. Prior use of lopinavir/ritonavir was found to be negatively associated with virological response to an enfuvirtide-containing regimen (22). However, lopinavir/ritonavir added as a new agent was associated with improved virological response (22), similar to the improved responses demonstrated in studies evaluating the addition of a new boosted PI (tipranavir/ritonavir in the
RESIST studies [39,40] and darunavir/ritonavir [TMC 114/r] in the POWER studies $[27,28]$ ).

Selection of concomitant agents is less problematic with enfuvirtide than with other antiretrovirals because drug interactions are unlikely to be an issue (19).

Recommendations for optimizing the background regimen:

- Use resistance testing along with expert interpretation to assist in the selection of optimal background therapy to accompany enfuvirtide. Review prior resistance test results to assess mutations that could re-emerge under selective drug pressure (IA).

- Use as many active drugs as possible concomitantly in the optimal background regimen, including new boosted PIs (IA).

\section{Therapeutic drug monitoring and enfuvirtide dosing}

Therapeutic drug monitoring currently does not appear to have a role in enfuvirtide treatment, but could be a useful tool in optimizing the efficacy of enfuvirtide-containing regimens in multidrug-experienced patients. The trough concentration of enfuvirtide after 12 weeks of treatment has been found to be associated with virological suppression at week 12 (59). More research is needed to clarify the potential role of enfuvirtide plasma level monitoring.

Preliminary data have not shown a difference in response to enfuvirtide based on body mass index (60). Although enfuvirtide's pediatric dosage is adjusted by weight, it remains unclear whether enfuvirtide dose should be adjusted for weight in adults.

\section{Use of 12 -week response to enfuvirtide in treatment decisions}

In the TORO studies, treatment-experienced patients who had good virological and immunological responses to enfuvirtide therapy at week 12 (eg, a decrease of at least $1 \log _{10}$ HIV-1 RNA copies $/ \mathrm{mL}$ and an increase of at least $50 \mathrm{CD} 4$ cells $/ \mu \mathrm{L}$, respectively) were very likely to maintain or improve their responses up to week 96 (61). Even though patients without a strong week 12 virological response were unlikely to achieve undetectable viral load, many of these patients who had a good week 12 immunological response had some immune recovery at week 96.

Recommendation for the use of 12-week response to enfuvirtide:

- Evaluate enfuvirtide response at 12 weeks to assess the likelihood of long-term response. A positive response to enfuvirtide after 12 weeks of treatment indicates a greater likelihood of a long-term response. However, a lack of virological response at 12 weeks does not rule out a positive long-term immunological outcome; therefore, evaluation at 12 weeks should include virological, clinical and immunological outcomes (IB).

\section{Enfuvirtide discontinuation}

In patients with virological failure despite HAART, there may be an advantage to continuing a failing regimen if it impairs viral fitness and slows immunological deterioration (62). In a study by Morse et al (63), more new mutations resulting in antiretroviral resistance were found in patients treated with 
enfuvirtide with rising viremia than in those with persistent low-level viremia. Some evidence indicates that enfuvirtide may impair viral fitness $(37,38,64)$. In a randomized trial of patients who achieved virological suppression for more than nine months with an optimized regimen containing enfuvirtide, the risk of viral rebound was less in those who remained on enfuvirtide versus those who discontinued enfuvirtide (65).

\section{Recommendation for enfuvirtide discontinuation:}

- The decision to discontinue enfuvirtide if there is lack of virological suppression or rebound following exposure should be considered in the context of the entire antiretroviral regimen and the immunological response (IIIA).

\section{PATIENT EDUCATION AND SUPPORT}

The key to the optimal use of enfuvirtide is good patient education and support at all levels, including physicians, nurses, pharmacists, families and friends.

\section{Initiation of enfuvirtide therapy}

The patient and the physician need to be ready and motivated to use enfuvirtide. The patient should understand the need for enfuvirtide, the reasons for use at this point in his/her therapy, the likelihood of its effectiveness, and the time commitment for preparation and administration.

Before initiating enfuvirtide treatment, issues of patient anxiety about needles and injection site reactions need to be considered, along with the risk of triggering relapses in recovering drug addicts. A negative, previous personal or family experience with injections may be overcome with the use of a needle-free injection system such as the Biojector (Bioject Inc, USA). Concerns about self-injection have been found to decrease with Biojector use $(66,67)$. Biojector has also been found to reduce injection site reactions, as well as pain immediately after injection $(66,68)$.

The patient should be made fully aware of the symptoms and expected duration of injection site reactions, and strategies for the prevention and management of injection site reactions should be reviewed, including injection technique and location. Rotation of injection sites, a smaller gauge needle (31G), or a needle-free injection system may prove useful in minimizing injection site reactions $(66,68,69)$. Some patients using standard needles have reported that slow injection, massaging of the site, and the use of hot or cold compresses may lessen reactions (18). In addition, recognition of signs of local infection should be reviewed. Allergic reactions, although rare, should be discussed. Successful desensitization of an enfuvirtideinduced skin hypersensitivity reaction has been reported (70).

The impact of enfuvirtide administration on daily activities, such as work, social life, travel and privacy, should be addressed with the patient. The effect of injection site reactions on physical appearance may also need to be addressed.

Some patients may have physical limitations that restrict their ability to administer enfuvirtide injections; therefore, the following should be assessed:

- adequacy of vision;

- the ability to use both hands, including issues of strength and tremors;

- mental capacity and memory, specifically, the ability to learn and remember procedures;
- the presence of adequate subcutaneous tissue, as many treatment-experienced patients have subcutaneous fat wasting; and,

- the availability of resources to help with injections and provide psychological support (eg, caregiver, partner, friends, family, support groups, social worker and pharmacist).

The patient should be well educated about enfuvirtide reconstitution and injection technique. Sterile technique should be emphasized. Tools such as videos and patient literature may also be useful.

Special attention should be given to the prevention and management of accidental HIV exposure by the caregiver, if applicable. Caregivers should be counselled to seek medical attention immediately if a needle stick injury occurs. Accidental exposure guidelines are available (71). Correct techniques for the disposal of needles and syringes should be carefully explained and safe containers provided to prevent accidental exposure.

\section{Ongoing support}

Ongoing patient support is important for retention on enfuvirtide therapy $(72,73)$. A $24 \mathrm{~h}$ contact number of an experienced health care provider should be provided to the patient to address questions and problems.

The patient should be reassessed regularly, for example, weekly for one month, every two weeks in the second month of treatment, and then monthly. During these reassessments, the following should be reviewed:

- injection technique;

- side effects, including injection site reactions;

- adherence, including missed doses and reasons for missing a dose, as well as whether or not counselling should be provided;

- the patient's motivation to continue antiretrovirals and enfuvirtide; and,

- acceptance of treatment: ease of injection and impact on activities of daily living.

Physicians have an opportunity to improve lagging motivation in patients who have either a good virological or immunological response, or both, at week 12 , because these patients are likely to have long-term positive benefits from enfuvirtide therapy (61).

\section{Recommendations for patient education and support:}

- Assess the appropriateness of enfuvirtide therapy for the individual patient, taking into account issues such as readiness and motivation, anxiety, physical issues that may limit their ability to administer the drug, and the availability of resources to help with injections and psychological support (IIIA).

- Educate the patient (and caregiver, if necessary) thoroughly on injection procedures, including reconstitution, injection technique, disposal of needles and syringes, and drug storage (IIIA).

- Counsel the patient on side effects, including injection site reaction prevention and management, and allergic reactions (IIIA). 
TABLE 3

Balance of benefits and barriers in the use of enfuvirtide

\begin{tabular}{ll}
\hline Potential benefits & Potential barriers \\
\hline Decreased viral load & Subcutaneous self-injection \\
Increased CD4 cell count & Injection site reactions \\
Fewer gastrointestinal toxicities & Impact on daily routine \\
Lack of hepatic or other significant & Privacy/travel issues \\
$\quad$ laboratory toxicities & Cost/payer coverage \\
Clinical improvements & \\
Absence of drug-drug interactions & \\
Improved quality of life & \\
\hline
\end{tabular}

- Provide ongoing support and regular reassessment (IIIA).

- Provide appropriate documentation to assist the patient in transporting injection supplies during air or international travel (IIIB).

\section{CONCLUSIONS}

Enfuvirtide is a first-in-class drug that targets HIV by inhibiting viral fusion with the host cell. It is a useful addition to antiHIV therapeutic strategies. Thus far, it has provided antiviral, immunological and clinical benefits, has a low toxicity, and contributes to improved quality of life in patients with HIV. The route of administration of this drug (subcutaneous injection) represents a new challenge for HIV/AIDS patients and their treating physicians, but these challenges can be overcome.

Ultimately, the benefits of enfuvirtide need to be balanced against the barriers (Table 3). It is essential that physicians be able to identify patients who are likely to benefit the most from the drug at an optimal time in treatment, rather than waiting until the late stages of the disease, when the benefits are likely to be limited. The present guidelines are intended to assist the treating physician in the appropriate selection and management of patients for the optimal use of enfuvirtide.

ACKNOWLEDGEMENTS: The workshop and the development of these guidelines were supported by an unrestricted grant from Hoffmann-La Roche Canada Inc. The authors wish to thank the following physicians for their comments on the guidelines: Gordon Arbess, David Fletcher, John Goodhew, Shariq Haider, Don Kilby, Alex Klein, Colin Kovacs, Mona Loutfy, Barry Merkley, Gary Rubin, Irving Salit, Michael Silverman, Jean-Guy Baril, Pierre Côté, Serge Dufresne, Normand Lapointe, Roger LeBlanc, John MacLeod, Danièle Rouleau, Réjean Thomas, Benoit Trottier, Sylvie Trottier, Mark Wainberg, Susan Burgess, Joss De Wet, Silvia Guillemi, Michael Madsen, Carol Murphy, Philip Sestak and Richard Taylor. The authors also wish to thank Ursula Jorch for editorial assistance.

\section{REFERENCES}

1. Clotet B, Raffi F, Cooper D, et al. Clinical management of treatment-experienced, HIV-infected patients with the fusion inhibitor enfuvirtide: Consensus recommendations. AIDS 2004;18:1137-46.

2. Mocroft A, Ledergerber B, Katlama C, et al; EuroSIDA study group. Decline in the AIDS and death rates in the EuroSIDA study: An observational study. Lancet 2003;362:22-9.

3. Walensky R, Paltiel A, Losina E, et al. Two million years of life saved: The survival benefits of AIDS therapy in the United States. In: 12th Conference on Retroviruses and Opportunistic
Infections, February 22 to 25, 2005, Boston, Massachusetts (abstract 143LB).

4. Panel on Clinical Practices for Treatment of HIV Infection. Guidelines for the Use of Antiretroviral Agents in HIV-1-Infected Adults and Adolescents. <www.aidsinfo.nih.gov/guidelines> (Version current at June 5, 2006).

5. Yeni PG, Hammer SM, Hirsch MS, et al. Treatment for adult HIV infection: 2004 recommendations of the International AIDS Society USA Panel. JAMA 2004;292:251-65.

6. Rinehart A, Lecocq P, Pattery T, Wasikowsi B, Bacheler L. Evolution in the diversity of HIV-1-resistance mutation patterns in $>128,000$ clinical samples received for resistance analysis from 1998 to 2004. In: 12th Conference on Retroviruses and Opportunistic Infections, February 22 to 25, 2005, Boston, Massachusetts (abstract 684).

7. Lohse N, Obel N, Kronborg G, et al. Declining risk of triple-class antiretroviral drug failure in Danish HIV-infected individuals. AIDS 2005;19:815-22.

8. Lampe F, Gatell J, Staszewski S, et al. Trends over time in initial virological failure of first HAART: 1996 to 2002. A joint cohort analysis of 4143 subjects. In: 12th Conference on Retroviruses and Opportunistic Infections, February 22 to 25, 2005, Boston, Massachusetts (abst 593).

9. Bartlett J, Fath M, DeMasi R, et al. An updated meta-analysis of triple combination therapy in antiretroviral-naive HIV-infected adults. In: 12th Conference on Retroviruses and Opportunistic Infections, February 22 to 25, 2005, Boston, Massachusetts (abst 586).

10. Hogg RS, Bangsberg D, Alexander C, et al. Drug resistance is associated with an increased risk of death in patients first starting HAART. In: 12th Conference on Retroviruses and Opportunistic Infections, February 22 to 25, 2005, Boston, Massachusetts (abst 712).

11. Little SJ, Holte S, Routy JP, et al. Antiretroviral-drug resistance among patients recently infected with HIV. N Engl J Med 2002;347:385-94.

12. Markowitz M, Mohri H, Mehandru S, et al. Infection with multidrug resistant, dual-tropic HIV-1 and rapid progression to AIDS: A case report. Lancet 2005;365:1031-8.

13. Vandamme AM, Sonnerborg A, Ait-Khaled M, et al. Updated European recommendations for the clinical use of HIV drug resistance testing. Antivir Ther 2004:9:829-48.

14. Harrigan PR, Wynhoven B, Brumme ZL, et al. HIV-1 drug resistance: Degree of underestimation by a cross-sectional versus a longitudinal testing approach. J Infect Dis 2005;191:1325-30.

15. Loutfy MR, Walmsley SL, Mullin CM, Perez G, Neaton JD; Terry Beirn Community Programs for Clinical Research on AIDS; Canada HIV Trials Network. CD4(+) cell count increase predicts clinical benefits in patients with advanced HIV disease and persistent viremia after 1 year of combination antiretroviral therapy. J Infect Dis 2005;192:1407-11.

16. Montaner JS, Harris M, Harrigan R, Hogg R, Wood E. A compromise strategy for patients with multiple drug failure. AIDS 2001;15:2470.

17. Matthews T, Salgo M, Greenberg M, Chung J, DeMasi R, Bolognesi D. Enfuvirtide: The first therapy to inhibit the entry of HIV-1 into host CD4 lymphocytes. Nat Rev Drug Discov 2004;3:215-25.

18. Product monograph, Hoffmann-La Roche Limited. FUZEON (enfuvirtide) for injection single-use vials: $108 \mathrm{mg} /$ vial. Antiviral Agent (HIV-1 Fusion Inhibitor).

19. Zhang X, Lalezari JP, Badley AD, et al. Assessment of drug-drug interaction potential of enfuvirtide in human immunodeficiency virus type 1-infected patients. Clin Pharmacol Ther 2004;75:558-68.

20. Lalezari JP, Henry K, O'Hearn M, et al. Enfuvirtide, an HIV-1 fusion inhibitor, for drug-resistant HIV infection in North and South America. N Engl J Med 2003;348:2175-85. (Erratum in 2003;349:1100).

21. Lazzarin A, Clotet B, Cooper D, et al; TORO 2 Study Group. Efficacy of enfuvirtide in patients infected with drug-resistant HIV-1 in Europe and Australia. N Engl J Med 2003;348:2186-95.

22. Nelson M, Arasteh K, Clotet B, et al. Durable efficacy of enfuvirtide over 48 weeks in heavily treatment-experienced

HIV-1-infected patients in the T-20 versus optimized background regimen only 1 and 2 clinical trials. J Acquir Immune Defic Syndr 2005;40:404-12.

23. Arasteh K, Lazzarin A, Clotet B, et al. TORO: 96 week virological and immunological response and safety evaluation of enfuvirtide with an optimized background regimen. Conference Abstract number MoOrB1058. Presented at the XV International AIDS Conference, July 11 to 16,2004 , Bangkok, Thailand. 
24. Cohen C, DeMasi R, Greenberg M, et al. Selection of nonenfuvirtide (ENF) vs. ENF-containing regimens leads to higher failure rates and loss of future antiretroviral (ARV) treatment options (poster H-580). Presented at the 44th Interscience Conference on Antimicrobial Agents and Chemotherapy, October 30 to November 2, 2004, Washington, DC.

25. Hicks C. RESIST-1: A phase 3, randomized, controlled, open label multicenter trial comprising tipranavir/ritonavir (TPV/r) to an optimized comparator protease inhibitor/r $(\mathrm{CPI} / \mathrm{r})$ regimen in antiretroviral (ARV) experienced patients: 24 week data. In: Program and abstracts of the 44th Interscience Conference on Antimicrobial Agents and Chemotherapy, October 30 to November 2, 2004, Washington, DC. (abst H-1137a)

26. Cahn P, Hicks C. RESIST-1 (R-1) and RESIST-2 (R-2) 48-week meta-analyses demonstrate superiority of protease inhibitor (PI) tipranavir+ritonavir (TPV/R) over an optimized comparator PI (CPR/R) regimen in antiretroviral (ARV) experienced patients. In: Program and abstracts of the 10th European AIDS Conference, November 17 to 20, 2005, Dublin, Ireland. (abst LBPS3/8)

27. Katlama C, Carvalho MT, Cooper D, et al. TMC114/r outperforms investigator-selected $\mathrm{PI}(\mathrm{s})$ in 3-class-experienced patients: Week 24 primary efficacy analysis of POWER 1 (TMC114-C213). Presented at the 3rd IAS Conference on HIV Pathogenesis and Treatment, July 24 to 27, 2005, Rio de Janeiro, Brazil. (poster WeOaLB0102)

28. Wilkin T, Haubrich R, Steinhart CR, et al. TMC114/r superior to standard of care in 3-class-experienced patients: 24-wks primary analysis of the POWER 2 study (C202). In: Program and abstracts of the 45th Interscience Conference on Antimicrobial Agents and Chemotherapy, December 16 to 19, 2005, Washington, DC. (abst H-413)

29. Trottier B, Walmsley S, Reynes J, et al. Safety of enfuvirtide in combination with an optimized background of antiretrovirals in treatment-experienced HIV-1-infected adults over 48 weeks. J Acquir Immune Defic Syndr 2005;40:413-21.

30. Cooper DA, Reiss P, Henry K, et al. The effects of enfuvirtide therapy on body composition and serum lipids through 48 weeks in the TORO trials. Presented at the 11th Conference on Retroviruses and Opportunistic Infections, February 8 to 12, 2004, San Francisco, California. (poster number 715)

31. Walmsley S, Henry K, Katlama C, et al. Enfuvirtide (T-20) crossreactive glycoprotein 41 antibody does not impair the efficacy or safety of enfuvirtide. J Infect Dis 2003;188:1827-33.

32. Sista PR, Melby T, Greenberg ML, et al. Subgroup analysis of baseline susceptibility and early virological response to enfuvirtide in the combined TORO studies. Antivir Ther 2003;8:S60.

33. Sista PR, Melby T, Davison D, et al. Characterization of determinants of genotypic and phenotypic resistance to enfuvirtide in baseline and on-treatment HIV-1 isolates. AIDS 2004;18:1787-94

34. Su C, Heilek-Snyder G, Ravindran P, et al. Substitutions within HIV gp41 amino acids 36-45 are identified as the primary determinants for loss of in vitro susceptibility to enfuvirtide: Results of data mining analyses of genotypic changes in gp41 in TORO 1 and TORO 2 that associate with changes in phenotypic susceptibility to enfuvirtide. Presented at the XIII International HIV Drug Resistance Workshop, June 8 to 12, 2004, Canary Islands, Spain. (poster number 162)

35. Loutfy M, Montaner J, Raboud J, et al. Genotypic resistance assay for entire gp-41 sequence with identification of gp-41 polymorphisms in enfuvirtide-naïve patients and new gp-41 mutations in patients failing enfuvirtide. In: Program and abstracts of the XV International AIDS Conference, July 11 to 16, 2004, Bangkok, Thailand. (abst WeOrB1292)

36. Greenberg ML, Cammack N. Resistance to enfuvirtide, the first HIV fusion inhibitor. J Antimicrob Chemother 2004;54:333-40.

37. Deeks S, Lu J, Hoh R, et al. Interruption of enfuvirtide in patients with enfuvirtide resistance. In: Program and abstracts of the 12th Conference on Retroviruses and Opportunistic Infections, February 22 to 25, 2005, Boston, Massachusetts. (abst 680)

38. Lu J, Sista P, Giguel F, Greenberg M, Kuritzkes DR. Relative replicative fitness of human immunodeficiency virus type 1 mutants resistant to enfuvirtide (T-20). J Virol 2004;78:4628-37.

39. Cooper D, Hicks C, Cahn P, et al. 24-Week RESIST Study Analyses: The efficacy of tipranavir/ritonavir is superior to lopinavir/ritonavir, and the TPV/r treatment response is enhanced by inclusion of genotypically active antiretrovirals in the optimized background regimen. In: Program and abstracts of the $12 \mathrm{th}$
Conference on Retroviruses and Opportunistic Infections, February 22 to 25, 2005, Boston, Massachusetts. (abst 560)

40. Valdez $\mathrm{H}$, McCallister S, Kohlbrenner V, et al. Tipranavir/ritonavir (TPV/r) $500 \mathrm{mg} / 200 \mathrm{mg}$ BID drives week 24 viral load (VL) below 400 copies $/ \mathrm{mL}$ when combined with a second active drug (T-20) in protease inhibitor experienced HIV+ patients. In: Program and abstracts of the 3rd IAS Conference on HIV Pathogenesis and Treatment, July 24 to 27, 2005, Rio de Janeiro, Brazil. (abst WeOa0205)

41. Montaner J, Guimaraes D, Chung J, Gafoor Z, Salgo M, DeMasi R. Prognostic staging of extensively pretreated patients with advanced HIV-1 disease. HIV Clin Trials 2005;6:281-90.

42. Walmsley S, Clotet B, Cooper D, et al. Efficacy of enfuvirtide in subgroups of patients through 48 weeks of therapy in the TORO trials. Presented at the 9th European AIDS Conference, October 25 to 29, 2003, Warsaw, Poland. (poster number 7.3/15)

43. Ruiz L, Romeu J, Martinez-Picado J, et al. Selection of drugresistance mutations in chronic HIV-infected patients during therapy Interruptions guided by CD4+ T-cell counts and viral load levels: The Tibet Study. In: Program and abstracts of the 12th Conference on Retroviruses and Opportunistic Infections, February 22 to 25, 2005, Boston, Massachusetts. (abst 679)

44. Hatano H, Hunt P, Weidler J, et al. Rate of viral evolution and risk of losing future drug options in heavily pre-treated patients remaining on a stable partially suppressive regimen. In: Program and abstracts of the 13th Conference on Retroviruses and Opportunistic Infections, February 5 to 6, 2006, Denver, Colorado. (abst 615)

45. Church JA, Hughes M, Chen J, et al; Pediatric AIDS Clinical Trials Group P1005 Study Team. Long term tolerability and safety of enfuvirtide for human immunodeficiency virus 1-infected children. Pediatr Infect Dis J 2004;23:713-18.

46. Bellibas SE, Siddique Z, Dorr A, et al; T20-310/NV16056 Study Group. Pharmacokinetics of enfuvirtide in pediatric human immunodeficiency virus 1 -infected patients receiving combination therapy. Pediatr Infect Dis J 2004;23:1137-41.

47. Bourgarit A, Lascoux C, Palmer P, et al. First-line use of enfuvirtide-containing HAART regimen with dramatic clinical and immunological improvement in three cases. AIDS 2006;20:471-3.

48. Miralles GD, Dolker M, Thommes J, et al. Effect of treatment interruptions on estimation of baseline (BL) antiretroviral (ARV) susceptibility and subsequent virological response in treatmentexperienced patients. Presented at the 7th International Congress on Drug Therapy in HIV Infection, November 14 to 18, 2004, Glasgow, United Kingdom. (poster number P17)

49. Beatty G, Lu J, Hunt P, et al. Randomized pilot study of immediate enfuvirtide-based therapy vs. a treatment interruption followed by enfuvirtide-based therapy in highly treatment-experienced patients. In: Program and abstracts of the 12th Conference on Retroviruses and Opportunistic Infections, February 22 to 25, 2005, Boston, Massachusetts. (abstract 581)

50. Baxter JD, Mayers DL, Wentworth DN, et al. A randomized study of antiretroviral management based on plasma genotypic antiretroviral resistance testing in patients failing therapy. CPCRA 046 Study Team for the Terry Beirn Community Programs for Clinical Research on AIDS. AIDS 2000;14:F83-93.

51. Durant J, Clevenbergh P, Halfon P, et al. Drug-resistance genotyping in HIV-1 therapy: The VIRADAPT randomised controlled trial. Lancet 1999;353:2195-9. (Erratum in 1999;354:1128).

52. Tural C, Ruiz L, Holtzer C, et al. Clinical utility of HIV-1 genotyping and expert advice: The Havana trial. AIDS 2002;16:209-18.

53. Hirschel B. A randomized trial assessing the impact of phenotypic resistance testing on antiretroviral therapy, by Cohen et al. AIDS 2003;17(Suppl 4):S114-6.

54. Wegner SA, Wallace MR, Aronson NE, et al. Long-term efficacy of routine access to antiretroviral-resistance testing in HIV type 1infected patients: Results of the clinical efficacy of resistance testing trial. Clin Infect Dis 2004;38:723-30.

55. Perez-Elias MJ, Garcia-Arota I, Munoz V, et al. Phenotype or virtual phenotype for choosing antiretroviral therapy after failure: A prospective, randomized study. Antivir Ther 2003;8:577-84.

56. De Meyer S, Hill A, De Baere I, et al. Effect of baseline susceptibility and on-treatment mutations on TMC114 and control PI efficacy: Preliminary analysis of data from PI-experienced patients from POWER 1 and POWER 2. In: Program and abstracts of the 13th 
Conference on Retroviruses and Opportunistic Infections, February 5 to 6, 2006, Denver, Colorado. (abst 157)

57. Henry K, Lalezari M, O'Hearn M, et al. Enfuvirtide (T-20) in combination with an optimized background (OB) regimen alone in patients with prior experience or resistance to each of the three classes of approved antiretrovirals (ARVs) in North America and Brazil. In: Program and abstracts of the XIV International AIDS Conference, July 7 to 12 , 2002, Barcelona, Spain. (abst LbOr19B)

58. Schapiro J, Cahn P, Trottier B, et al. Effect of baseline genotype on response to tipranavir/ritonavir (TPV/r) compared with standard-ofcare comparator $(\mathrm{CPI} / \mathrm{r})$ in treatment-experienced patients: The Phase 3 RESIST- 1 and -2 Trials. In: Program and abstracts of the 12th Conference on Retroviruses and Opportunistic Infections, February 22 to 25, 2005, Boston, Massachusetts. (abst 104)

59. Bonora S, Gonzalez de Requena D, Castagna A, et al. Pharmacokinetic and pharmacodynamic determinants of virological response to enfuvirtide-based regimens. In: Program and abstracts of the 12th Conference on Retroviruses and Opportunistic Infections, February 22 to 25, 2005, Boston, Massachusetts. (abst 643)

60. Zhang X, Buss N, Salgo M, et al. Enfuvirtide pharmacokineticpharmacodynamic (PK-PD) relationship. Presented at the 9th European AIDS Conference, October 25 to 29, 2003, Warsaw, Poland. (poster number 4.1/4)

61. Raffi F, Katlama C, Saag M, et al. Week-12 response to therapy as a predictor of week 24, 48 and 96 outcome in patients receiving the HIV fusion inhibitor enfuvirtide in the T-20 versus optimized regimen only (TORO) trials. Clin Infect Dis 2006;42:870-7.

62. Deeks SG, Wrin T, Liegler T, et al. Virologic and immunologic consequences of discontinuing combination antiretroviral-drug therapy in HIV-infected patients with detectable viremia. N Engl J Med 2001;344:472-80.

63. Morse C, Maldarelli F, Dewar R, et al. Limited evolution of drug resistance in HIV-1 infected patients with persistent low-level viremia on a stable antiretroviral regimen for greater than one year. Presented at the 12 th Conference on Retroviruses and Opportunistic Infections, February 22 to 25, 2005, Boston, Massachusetts. (poster number 678)

64. Menzo S, Castagna A, Monachetti A, et al. Resistance and replicative capacity of HIV-1 strains selected in vivo by long term enfuvirtide treatment. New Microbiol 2004;27(2 Suppl 1):51-61.

65. Bonjoch A, Puig J, Miranda C, et al. Discontinuation of T-20 in HIV-infected patients on long lasting viral suppression. In: Program and abstracts of the 45th Interscience Conference of Antimicrobial
Agents and Chemotherapy, December 16 to 19, 2005, Washington, DC. (abst H-527)

66. Harris M, Larsen G, Valyi M, et al. Early experience in the use of the Bioject Needle-free Injection System (Biojector) for the Delivery of T20 (enfuvirtide) in treatment-experienced patients. Presented at the HIV DART 2004, December 12 to 16, 2004, Montego Bay, Jamaica. (poster number 063)

67. Gallant JE, Mackowiak JI, Dusek AC, Falcon RW, DeMasi R. Change in injection attitudes and patient-reported outcomes among patients with enfuvirtide (ENF) experience. In: Program and abstracts of the 3rd IAS Conference on HIV Pathogenesis and Treatment, July 24 to 27, 2005, Rio de Janeiro, Brazil. (abst WePe12.9C13)

68. Harris M, Joy R, Larsen G, et al. Enfuvirtide plasma levels and injection site reactions using a needle-free gas-powered injection system (Biojector). AIDS 2006;20:719-23.

69. True AS, Luo R, DeMasi R, Falcon R. An evaluation of HIV patient quality of life and tolerability after administration of enfuvirtide-based HAART using a smaller needle in a community practice setting. In: Program and abstracts of the 3rd IAS Conference on HIV Pathogenesis and Treatment, July 24 to 27, 2005, Rio de Janeiro, Brazil. (abst WePe6.3C09)

70. Shahar E, Moar C, Pollack S. Successful desensitization of enfuvirtide-induced skin hypersensitivity reaction. AIDS 2005;19;451-2.

71. Centers for Disease Control and Prevention. Updated U.S. Public Health Service Guidelines for the Management of Occupational Exposures to HIV and Recommendations for Postexposure Prophylaxis. <www.cdc.gov/mmwr/preview/mmwrhtml/ rr5409a1.htm > (Version current at May 26, 2006).

72. Varsalone D, Swartz K, Mohammed D. An enfuvirtide empowerment program: Do Not Enter. Presented at the 7th International Congress on Drug Therapy in HIV Infection, November 14 to 18, 2004, Glasgow, United Kingdom. (poster number P210)

73. Goodly J, DeMasi R, Khoo K, Mazikewich A, Thommes J. Optimal adherence to enfuvirtide achieved in the US Early Access Program. Presented at the 7th International Congress on Drug Therapy in HIV Infection, November 14 to 18 , 2004, Glasgow, United Kingdom. (poster number P130)

74. Gross PA, Barrett TL, Dellinger EP, et al. Purpose of quality standards for infectious diseases. Infectious Diseases Society of America. Clin Infect Dis 1994;18:421. 


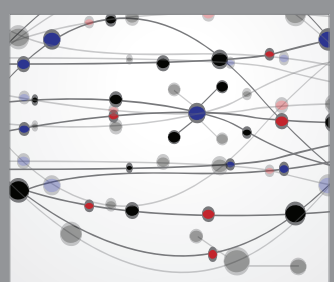

The Scientific World Journal
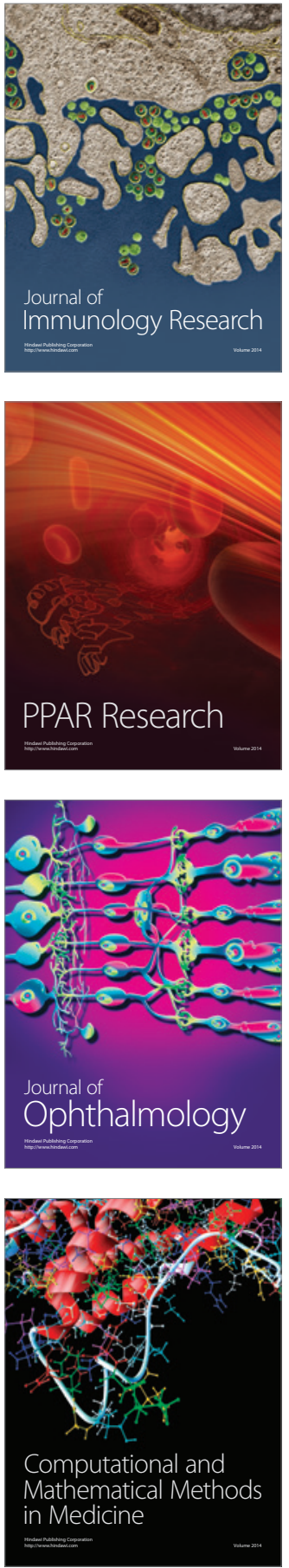

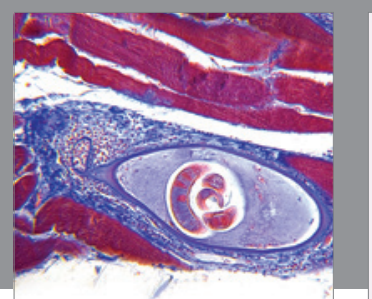

Gastroenterology Research and Practice

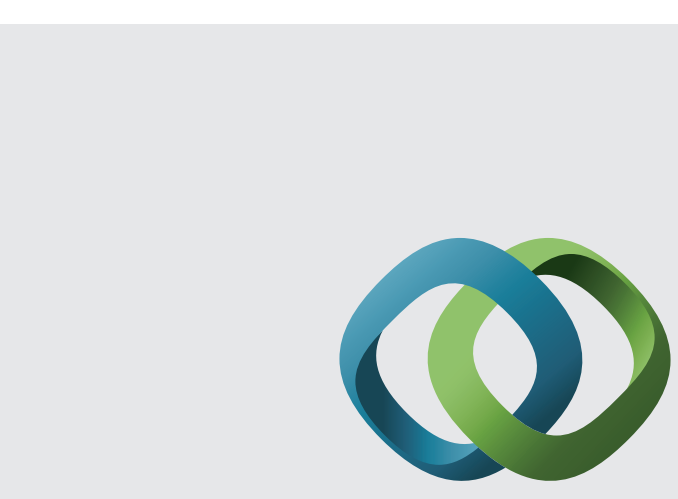

\section{Hindawi}

Submit your manuscripts at

http://www.hindawi.com
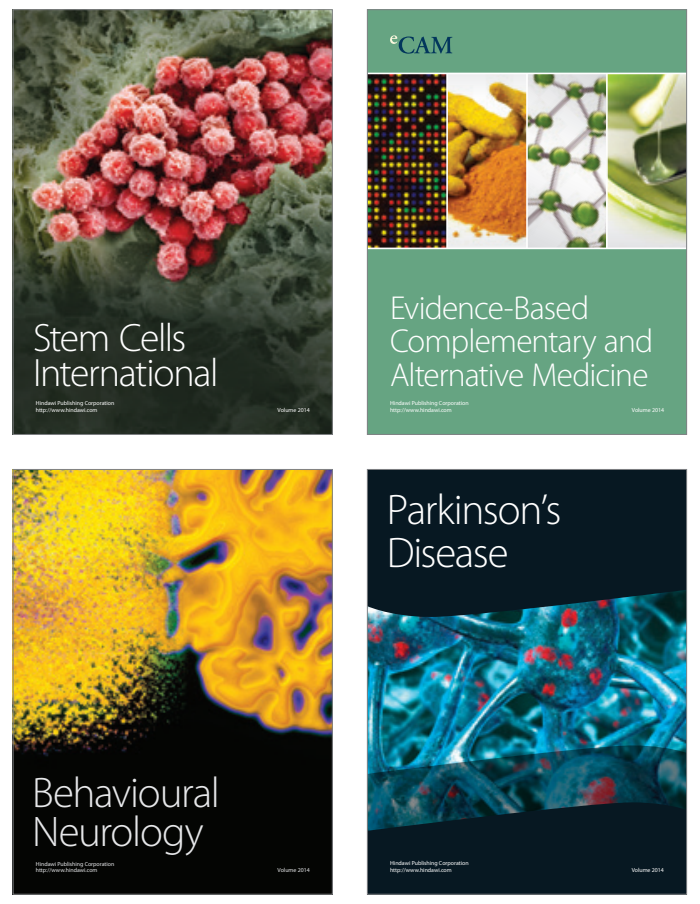
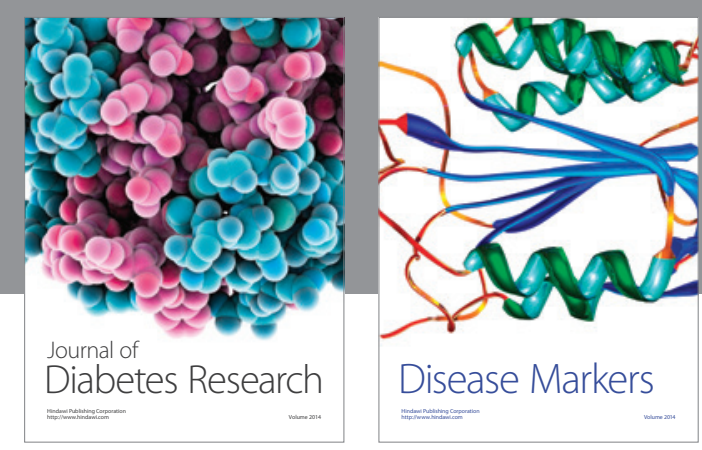

Disease Markers
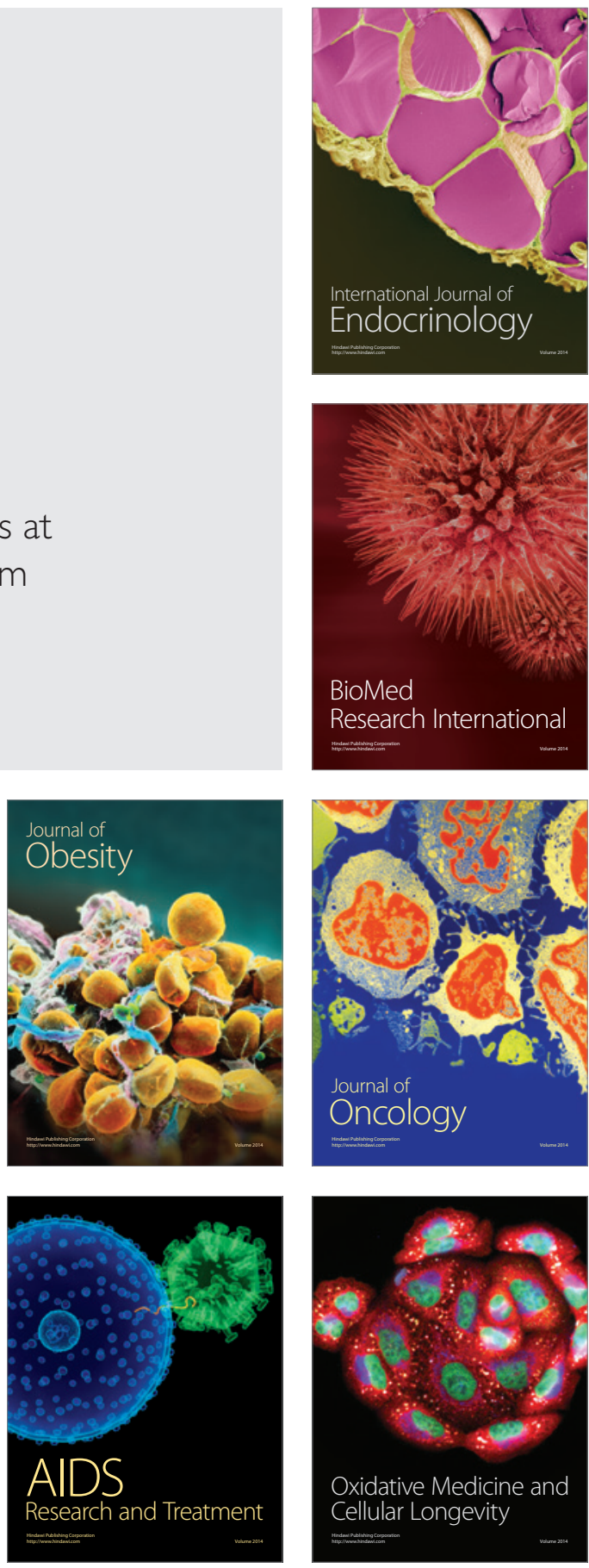